\title{
Interactive Simulation of Dynamic Crowd Behaviors using General Adaptation Syndrome Theory
}

\author{
Sujeong Kim \\ Stephen J. Guy \\ Dinesh Manocha \\ Ming C. Lin * \\ Department of Computer Science, UNC - Chapel Hill \\ http: / / gamma.cs.unc.edu/GAScrowd/
}

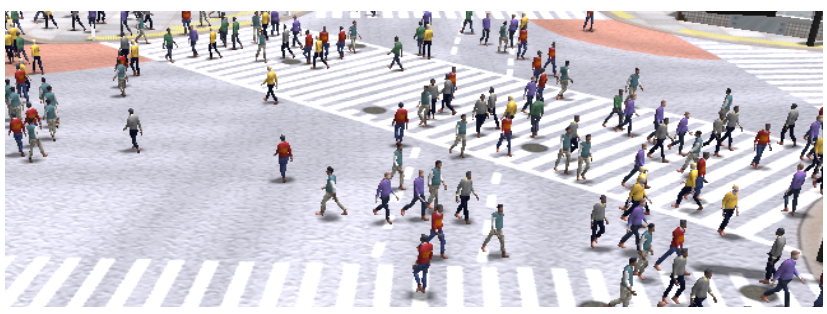

(a) Crossing immediately before light changes

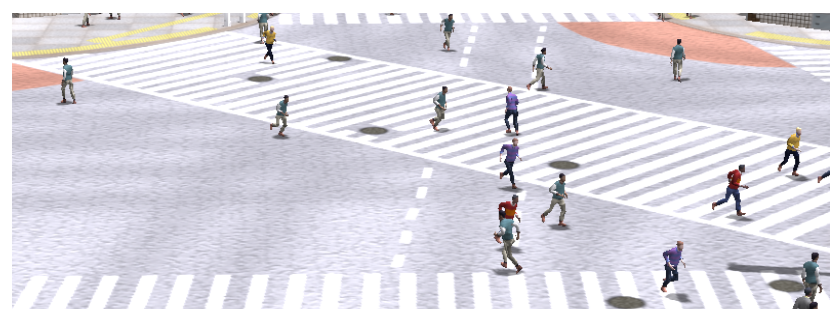

(b) Crossing after red light

Figure 1: Shibuya Crossing Scenario. A scenario simulating the scramble crossing near Shibuya metro station in Tokyo. (a) Agents start to walk quickly or jog when the walk signal begins flashing indicating little time left to cross. (b) When the light turns red, indicating no time left to cross safely, agents experience a high level of stress and run aggressively to cross as quickly as possible.

\begin{abstract}
We propose a new technique to simulate dynamic patterns of crowd behaviors using stress modeling. Our model accounts for permanent, stable disposition and the dynamic nature of human behaviors that change in response to the situation. The resulting approach accounts for changes in behavior in response to external stressors based on well-known theories in psychology. We combine this model with recent techniques on personality modeling for multiagent simulations to capture a wide variety of behavioral changes and stressors. The overall formulation allows different stressors, expressed as functions of space and time, including time pressure, positional stressors, area stressors and inter-personal stressors. This model can be used to simulate dynamic crowd behaviors at interactive rates, including walking at variable speeds, breaking laneformation over time, and cutting through a normal flow. We also perform qualitative and quantitative comparisons between our simulation results and real-world observations.
\end{abstract}

Keywords: crowd simulation, psychological models, dynamic behaviors

\section{Introduction}

Simulating the wide variety of behaviors seen in real-world crowds is important for many interactive applications, including games and virtual environments. There are no known computational models

\footnotetext{
*\{sujeong,sjguy,dm,lin\}@cs.unc.edu
}

that can simulate different types of crowd behaviors. At a broad level, crowd behaviors are governed by the characteristics of the individual humans and the surrounding environment.

Psychologists have extensively studied human characteristics and behaviors. Differences in human behaviors are governed by multiple factors, including differences in stimuli, genetic endowment, physiological state, cognitive state, social environment, cultural environment, previous life experiences, and personal characteristics [Eysenck 2002]. Despite this diversity, factors affecting human behaviors can be categorized into some basic types.

Attribution theory, for example, divides these causes into dispositional attributes and situational attributes. Dispositional attributes capture internal factors such as personality or characteristics, while situational attributes capture external factors such as current situation [Heider 1982]. Cattell [1952] suggested a similar divide in the causes of behavior termed personality and situational factors. Our proposed technique for simulating dynamic crowd behaviors is based on this dichotomy, and we use separate models for an agent's personality and another one to account for situational factors.

In crowd simulation literature, techniques to model heterogeneous behaviors using personality models have been proposed [Durupinar et al. 2011; Guy et al. 2011]. Resulting simulations can successfully generate a variety of behaviors happening in a scene, but may not be able to model dynamic behaviors. These dynamic behaviors correspond to changes in individual and crowd behaviors in response to a situation. For example, a calm or composed person walking through a pedestrian crossing may become aggressive when the light turns red from green. Similarly, the same person may cut through a crowd when a train approaches the platform at a train station. The dynamic nature of human and crowd behaviors is also observed during fearful or panicked situations, such as fire evacuations, where individuals change behaviors in response to emergencies and alarms.

Our objective is to model such dynamic crowd behaviors at interactive rates. We model these behaviors as a reaction to meet certain demands or cope with the changes in a situation or environment. These situational factors will be referred to as stressors, and the effect of these stressors on the agents will be measured as stress. Our 
approach is build on the psychological theory of General Adaptation Syndrome [Selye 1956] that provides a well-established behavior model of how humans react to stress.

Main Result: We present a new technique to simulate dynamic patterns of crowd behaviors by considering various types of stressors. Our model accounts for both stable, consistent aspects of behaviors, influenced by personality, and the dynamic changes in behavior due to situational factors. Our main contribution is a method that incorporates well-established psychological models of stress into crowd simulation. Our algorithm generates realistic, dynamicallychanging crowd behaviors based on a few high-level parameters that model how individuals vary in their response to stress.

We model stressors as functions of space and time that can generate various real-world crowd behaviors. These include prototypes of many common stressors based on time constraints, agent positions, and other environmental factors. These stressors can be used in conjunction with goal-directed, multi-agent simulations to easily generate a rich variety of behaviors. An agent's reaction to a stressor depends on both an agent's personality and the stressor itself. More specifically, we model the measured value of stress that a agent receives from stressors as a function of both (personality-dependent) internal weight and (situation-dependent) external weights.

The resulting simulation shows a variety of dynamic crowd behaviors under stress, such as cutting through the crowds, walking with variable speeds, and breaking lane-formation over time or in different situations. We perform both qualitative and quantitative comparison between our simulation results and real-world observations. Our method has a small computational overhead and can simulate thousands of agents responding to dynamic stressors in real time on a single-core CPU.

The rest of our paper is organized as follows. Section 2 provides an overview of related work both from crowd simulation and psychology. Section 3 gives an overview of psychological models and of our approach. Section 4 discusses our approach to modeling stress accumulation. Section 5 presents the overall dynamic crowd simulation algorithm. We highlight the results in Section 6.

\section{Related Work}

In this section, we give a brief overview of prior work from both psychology and crowd simulation literature. Our discussion here focuses on modeling dynamic behaviors due to stress. For a broader coverage of human psychology, we refer the reader to Eysenck [2002].

\subsection{Stress}

Several researchers have attempted to characterize how humans respond to stress in terms of both internal, physiological changes and external, behavioral changes. Early attempts to model how human behavior changes in different situations include the work of Cattell [1952], who proposed a mathematical formula to predict human behavior as a function of personality and situation. More recently, Leon [2010] have extended this work to the pedestrian behavior, modeling the increased aggression people exhibit when stressed.

Selye [1956] proposed a broad framework for understanding how the response to stress changes over time. His General Adaptation Syndrome theory presents a non-specific, non-unique model of stress response to stimuli. Since this work, other studies about stress in a broad aspect have been performed, investigating its relationship to general activities, physiological effects, emotional, behavior, and cognitive performance [Eriksen et al. 1999; Mordkoff
1964]. The connection between stress and aggression has been particularly well established [Evans 1984; Anderson 2001; K.B. and Rasmussen 1979] and holds across a variety of stressors [Berkowitz 1990; Miller 1941].

\subsection{Crowd Simulation}

The area of crowd simulation and multi-agent navigation is an active area of research, with a wide variety of methods and results. We primarily focus on interactive methods for modeling crowd behaviors. For a broader coverage of the field we refer the reader to a recent survey [Pelechano et al. 2008].

\subsubsection{Interactive Crowd Simulation}

There are several frameworks proposed for simulating and rendering large number of crowds. The Virtual Dublin project simulated crowds in an urban simulation at interactive frame rates [Dobbyn et al. 2005]. Yersin et al. [2009] proposed a method using precomputed crowd patches to populate a large-scale virtual environment for real-time simulations. Parallel GPU-based algorithms have also been proposed for both crowd simulation and high-quality rendering [Shopf et al. 2008].

Many real-time techniques have also been suggested for goaldirected multi-agent simulations. Among them the HiDAC system is able to simulate various behaviors [Pelechano et al. 2007]. Reciprocal Collision Avoidance based methods have been successfully applied to simulate crowds [van den Berg et al. 2011]. Patil et al. [2011] proposed an interactive algorithm based on navigation fields, where users can directly control the crowd movement.

\subsubsection{Behavior Modeling}

Rule-based approaches are commonly used to model complex behaviors. These include frameworks based on motor, perceptual, behavioral, and cognitive components for modeling pedestrian behavior [Shao and Terzopoulos 2005] and modeling decision-making process [Yu and Terzopoulos 2007].

Reynolds [1999] modeled flocking behaviors as those of individuals and pairs, by combined behavior as group behavior. Yeh et al. [2008] described the geometric notion of a composite agent, which can model different behaviors including aggression, social priority, authority, protection, and guidance.

Many researchers have used psychological factors in crowd simulation. Pelechano et al. [2005] simulated different wayfinding behaviors of trained/untrained leaders and the followers in emergency situations. These behavior patterns are chosen based on the given role, and may not change during simulation. Sakuma et al. [2005] proposed a local collision avoidance method that switches discreetly between smooth and urgent avoidance behaviors based on the urgency of collisions. Nygren [2007] proposed a system which models the effects of psychological factors by using artist derived rules to change behaviors when the agents are fearful, fatigued or happy.

More recently, there have been attempts to create realistic, heterogeneous crowd behavior based on human psychology, especially personality traits [Guy et al. 2011; Durupinar et al. 2011]. These approaches provide a way to model heterogeneous behaviors, but the behavior patterns do not change over time. Our approach builds on these works to model dynamic crowd behaviors. 


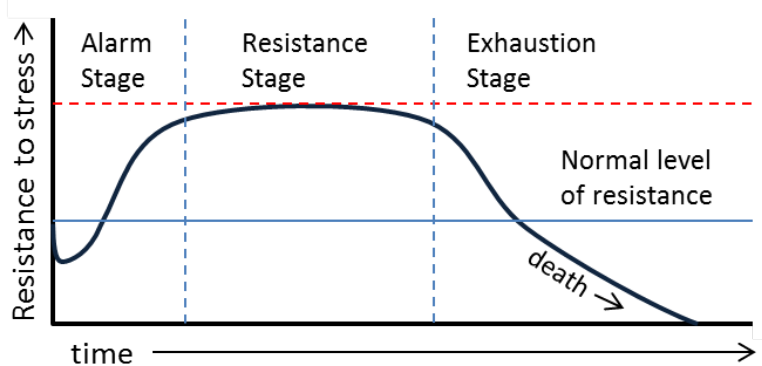

(a) Selye's General Adaptation Syndrome model

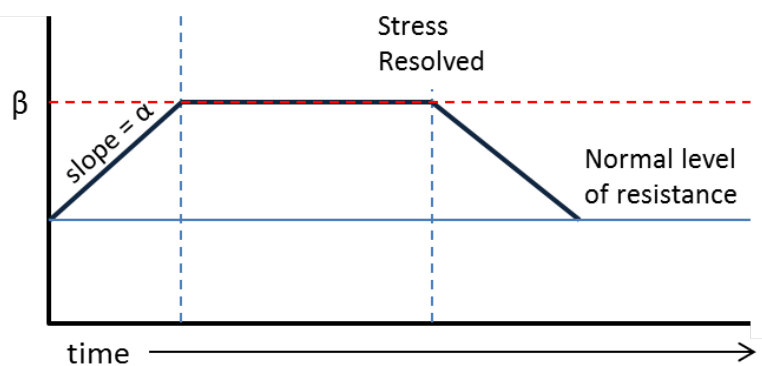

(b) Response generated by our model

Figure 2: General Adaptation Syndrome and approximation (a) GAS model of the human response to stress. After an initial disturbance, the resistance level increases up to maximum capacity. If the stress is chronic or unresolved, the resistance gets weaker and is no longer effective (causes illness or death). (b) Our approximation of GAS model. We assume acute stress, i.e. no exhaustion stage. Instead, agents are relieved from stress when the stress is resolved. The shape of the response is determined by the stress accumulation rate $\alpha$ and maximum capacity $\beta$

\section{Preliminaries and Overview}

In this section, we introduce some important concepts from psychology literature on modeling stress and highlight the link between stress and behaviors. We also give an overview of our method for applying this established psychological literature to multi-agent simulations for generating dynamic crowd behaviors.

\subsection{Psychological Models of Stress}

There are multiple definitions of stress in psychology literature. In a broad sense, stress can be defined as any change caused by interactions between the environment and individuals. Generally, stress is caused by a discrepancy between environmental demands and the abilities of individuals [Cox 1978]. In other words, people become stressed when they feel they are challenged or they need to cope with the current situation. Stressors are what cause the stress, they can be a situation, an object, or even other individuals. There are a number of sources that cause stress. In this paper, we focus on the following types of stressors:

1. loads given to individuals (challenging situations), e.g. time constraints associated with the goal of each agent;

2. perceived threats, e.g. fire, threatening animals or objects;

3. unpleasant events, e.g. heat, noise, air pollution (smoke, malodor), and over-crowding.

The emotional or behavioral effect of stress is generally associated with increased aggression. This link can be found is both psychological models of emotion [Berkowitz 1990] and empirical studies of human behavior [Evans 1984; Anderson 2001] However, the result of stress is not always negative. In some situations increased aggression can have positive effects, and can improve performance up to a point [Yerkes and Dodson 1908].

By measuring the connection between how people act (measured through recorded observation) and how people feel (measured indirectly via heart rate, skin temperature and self reporting) psychologists have established a consistent relationship between increased stress and increased aggressive and impulsive behavior [Anderson 2001]. This result has held across various stressors, different settings, cultures, and genders [Evans 1984; K.B. and Rasmussen 1979]. Our approach uses the result of these empirical studies to model various stressors and their effects as increasingly aggressive and impulsive crowd behaviors.

\subsection{General Adaptation Syndrome (GAS)}

To simulate the behavioral effects under a given value of stress, we use the General Adaptation Syndrome (GAS) formulation proposed by Hans Selye [1956]. Selye proposed the GAS model as a general response to any stressor (toxins, cold, injury, fatigue, fear, etc.) The GAS model has three stages of response: alarm, resistance, and exhaustion (see Figure 2a). When individuals perceive a stress, in the alarm stage, they ready themselves for "fight" or "flight". In the resistance stage, they work to resolve the stress at their full capacity. If the stressor is not removed (i.e. a chronic stressor), they reach the exhaustion stage and resistance becomes ineffective.

Research shows that the GAS model also applies to various physiological changes and general activities [Eriksen et al. 1999]. Additionally, there is a stable relationship between psychological response of how stress makes a person feel and the physiological response of how it changes their behavior [Mordkoff 1964].

\subsection{Approximation of the GAS Model}

While the GAS model suggests the shape of a person's stress responses, it does not provide quantitative values for the level of response to different stressors. We propose a quantitative approximation of the GAS model, which produces a stress response consistent with that model.

We first assume an agent is experiencing a perceived stress with a value of $\psi$. Our goal is to compute a stress response for an agent, denoted as $S$. This value will be a function of the perceived stress, $\psi$. To maintain consistency with the shape of the GAS response, our model has two main attributes. First, an agent's change in stress response is capped by a maximum rate, denoted as $\alpha$. This is to ensure that an agent's stress response does not jump suddenly in response to a sudden stress. Secondly, an agent's stress response is capped at some maximum amount, denoted as $\beta$. This is to ensure that if the perceived stress increases unboundedly, there will be a limit on an agent's response.

Taken together, $\alpha$ and $\beta$ will map the perceived stress $\psi$ to a stress response $S$ as follows:

$$
\frac{d S}{d t}= \begin{cases}\alpha & \text { if } \psi>S \\ \left\{-\alpha \leq \frac{d \psi}{d t} \leq \alpha\right\} & \text { if } \psi=S \\ -\alpha & \text { if } \psi<S\end{cases}
$$


where $S$ is capped at maximum of $\beta$. For efficiency reasons, we model $\alpha$ and $\beta$ as constants per agent per stressor, though, in general, these values can be a function of time or of the number of exposures to a stressor.

Figure $2 \mathrm{~b}$ shows the resulting stress response induced by a instantaneous, large value of $\psi$ (which lasts until the stress is resolved). This is similar to the stress induced by a sudden, loud warning alarm sounding. The stress response, which results from Eqn.1, shows a similar shape to that corresponding the GAS model (shown in Figure $2 a)$.

In general, an individual's stress response (parameterized by $\alpha$ and $\beta$ ) can vary between different people and across different situations. The values for these parameters can be chosen by an artist for a specific, potentially exaggerated effect (as discussed in Section 4.3) or chosen to match real-world data (as discussed in Section 5.3).

Our purpose is to provide a general framework that can simulate dynamically changing behavior triggered by stress response in realtime crowd simulations. To that end, we make some simplifying assumptions, notably, treating the parameters $\alpha$ and $\beta$ as constants, and a further assumption that agents will not be exposed to a stressor long enough to reach the exhaustion or death stage.

\subsection{Overview of our Approach}

Our overall system has three main components. Firstly, the stressor provides a source of stress for the agents. Secondly, the stress accumulation function Eqn.1 which is determined by the GAS model. Thirdly, a multi-agent simulation algorithm that is capable of changing an agent behavior by increasing its aggressiveness and impulsiveness.

The interaction between a stressor and an agent's perceived level of stress from that stressor is determined by the perceived stress function, $\psi$. The form of the perceived stress function varies based on the type of stress, and we highlight several examples in Section 4.1. The interactions between the agents are updated as a function of the accumulated stress, $S$. This aspect is discussed in Section 5. Figure 3 provides an overview of the system. In the next two sections we discuss details of how we model each component.

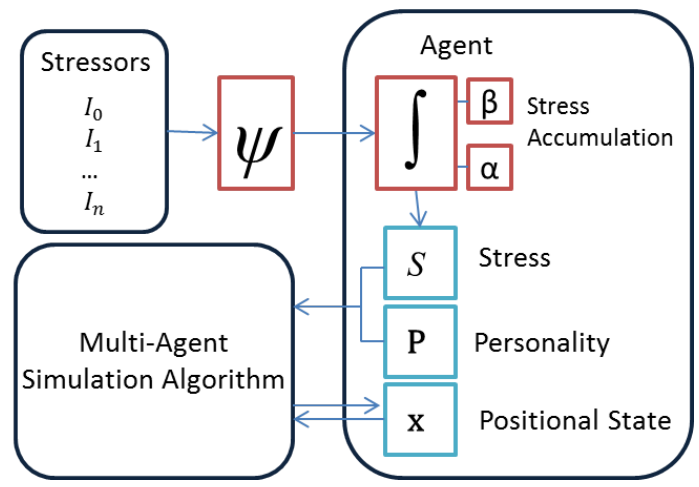

Figure 3: System Overview Different levels of stressed behaviors are simulated by updating agent parameters.

\section{Modeling Stress and Stressors}

In this section, we describe how we model stress in our simulator. We show how measurable physical quantities of a stressor are mapped to the perceived amount of stress and give specific examples for various prototype stressors.

\subsection{Perceived Stress}

In order to define the perceived amount of stress experienced by an agent, we adapt Stevens' psychophysical power law [Stevens 1957]. This law states the relationship between the perceived magnitude of a stress and the physical measurement of the stimulus intensity, e.g. the relationship between sound intensity and perceived loudness, luminance and perceived brightness, and density and perceived crowding.

Steven's Law states that the relationship between the perceived intensity of the stressor, $\psi$, and the magnitude of the physical intensity of the stressor, $I$, has the form:

$$
\psi(I)=k I^{n},
$$

with two parameters, a scale factor $k$ and an exponent $n$, which depend on the type of stressor. Stevens [1957] provides approximate exponent values for various stimulus. Additionally, the computation of the intensity $I$ also depends on the type of the stressor. Steven's Law was originally formulated for low level stressors, such as noise and heat. However, subsequent studies found that similar power laws can be applied to a wide range of stressors [Teghtsoonian and Frost 1982; Middlemist et al. 1976; Oswald and Bratfisch 1969]. Inpired by this observation, we use Eqn. 2 as a generic model of the effect of the stressors.

\subsection{Stressor Prototypes}

We define four different prototype stressors, which can be used to model a variety of forms of stress. These are time pressure, positional stressors, environmental stressors, and interpersonal stressors.

Time pressure: These are stressors associated with an attempt to reach a goal by a particular time. Examples could include crossing the street at a timed light, boarding a train before the doors close, or evacuating a building during a fire.

To model these type of stressors, agents are given a goal position and a time constraint of $t_{\text {allowed }}$. We model the intensity of the time pressure, $I_{t}$, as a function of the difference between the allowed time and the estimated arrive time. Formally:

$$
I_{t}=\max \left(t_{\text {estimated }}-t_{\text {allowed }}, 0\right),
$$

where $t_{\text {estimated }}$ is the estimated amount of time an agent will take to reach its goal, i.e. $t_{\text {estimated }}=$ distRemaing/avgSpeed.

Area stressors: These are stressors that arise from a condition in the environment. Examples include noise, heat, bright lights, and smoke. The intensity of these types of stressors is almost constant over a area, that is

$$
I_{a}= \begin{cases}c & \text { if } p_{a} \in A \\ 0 & \text { if } p_{a} \notin A\end{cases}
$$

where $A$ is the area under effect from the stressor and $p_{a}$ is the agent's current position.

Positional stressors: These are stressors associated with a localized source of stress. Unlike area stressors, these correspond to stressors whose intensity grows as an agent approaches them. Examples include both static stressors, such as fire, or dynamically moving stressors, such as a runaway car or an assailant.

Formally, we define the intensity as

$$
I_{p}=\left\|p_{a}-p_{s}\right\|,
$$


where $p_{a}$ and $p_{s}$ indicate the position of the agent and the stressor, respectively.

Some stressors, such as fire, have a high intensity over a large area. For these stressors, we use a Gaussian distribution with a standard deviation of $\sigma$ to compute the intensity:

$$
I_{p}=\mathcal{N}\left(p_{a}-p_{s}, \sigma\right)
$$

Interpersonal stressors: These are stressors associated with the stress coming from other agents. A common example includes crowding, where some people feel stress due to too many people being too close. These interpersonal stressors have been found to follow a similar exponential law [Middlemist et al. 1976; Oswald and Bratfisch 1969]. We model these stressors' intensity as a function of the difference between the preferred density of neighbors, and the actual density of neighbors:

$$
I_{i}=\max \left(n_{\text {cur }}-n_{\text {pref }}, 0\right) \text {, }
$$

where $n_{\text {cur }}$ is number of current neighbors in a unit space and $n_{\text {pref }}$ is the preferred number of neighbors in the same area.

\subsection{Stress Model}

Each of the above stressor prototypes define an intensity $I$ which, when combined with Eqn. (2), is used to define the perceived stress $\psi$. Given the current $\psi$, an agent's stress response, $S$, is determined by Eqn. (1).

The values of $\alpha$ and $\beta$ can be changed to control the stress response of an agent. The way people respond to stress varies based on the condition of body defense system and the coping style of the individual. In our model, agents with a high $\alpha$ can be thought of as tense and react very quickly to the introduction of a new stressor. Agents with a high $\beta$ react very intensely to stressors and show a much stronger reaction to the same stressor as compared to agents with a low $\beta$.

The values for $\alpha$ and $\beta$ can be chosen by the artist or scene designer to get a desired distribution of stress responses. Alternatively, these values can be determined from real-world data, as discussed in Section 5.3.

Multiple stressors: When exposed to multiple stressors, we compute a weighted sum of each stress value to find the total stress experienced by the agent. This model conforms with the discussion in Lazarus [1993] that people selectively pay attention to the stimulus.

Formally, we define the total perceived stress $s$ as

$$
S=\sum \omega_{i} S_{i}
$$

where $\omega_{i}$ is the weight of each individual stressor $S_{i}$. The values of $\omega_{i}$ can be chosen to weigh the stresses equally or can be used to give priority to more important stressors.

\section{Behavior Mapping}

In Section 4, we defined our psychologically-motivated model of how an agent's level of perceived stress, $S$, changes in response to various stressors. We now discuss how an agent's behavior changes in response to these changes in the stress level.

As discussed in Section 3.1, the primary observable response to increasing levels of stress is an increase in aggressive and impulsive behavior. Our method for modeling the dynamic changes in behavior that arise from stress relies on a multi-agent system capable of simulating changes in the levels of aggression and impulsiveness displayed by the agents. We use the multi-agent simulation algorithm proposed by Guy et al. [2011], though our methods could be easily applied to other approaches with similar capabilities.

\subsection{Incorporation of Behavior Changes}

Recently, Guy et al. [2011] proposed a method of reparameterizing the reciprocal-collision-avoidance based simulation method described in van den Berg et al. [2011] to achieve a perceptually based variation in agent behavior. The method was based on the results of a user study that asked participants to classify the apparent behavior of agents in terms of how aggressive, impulsive, shy, tense, active or assertive the agents appeared. The result of the study suggested a method for choosing simulation parameters that could achieve a desired agent behavior. These parameters include agent radius, preferred speed, planning horizon, number of neighbors, and agents sight distance. Different parameter values generate different goaldirected behaviors and local interaction with neighboring agents, which are perceived as personality.

They asserted that there are two primary dimensions (or principle components) of crowd behavior, and that these can be regarded as high-level parameters. The first, denoted PC1, was correlated to an increased level of of "extraverted" or more "intense" behavior from the agents. The second dimension, PC2, was associated with increasingly "careful" behavior. Using this parameterization, we are able to determine how to automatically select simulation parameters for the multi-agent modeling system to produce behaviors that appear to be as increasingly aggressive and impulsive. We denote this change in parameters as the stress behavior vector, $\mathbf{B}_{\text {stress }}$ since adding it to an agents current simulation parameters will increase their perceived level of stress. For the results in this paper, we use

$$
\mathbf{B}_{\text {stress }}=(\mathrm{PC} 1 \mathrm{PC} 2)\left(\begin{array}{c}
0.95 \\
-0.3
\end{array}\right)
$$

because it produces behavior which is predominately aggressive (very high "egocentricity") and somewhat impulsive (negative "carefulness").

\subsection{Coupling with Personality Attributes}

Guy et al. [2011] also provided a matrix, $A_{p c}$, which gives a linear mapping between the values of PC1 and PC2 and the simulation parameters. The same work further suggested a matrix, $A_{a d j}$ which maps a variety of different personality descriptors to simulation parameters. We determine the final behaviors of our agents as a linear combination of these two parameter matrices: the first representing an agent's situational response and the second the agent's stable personality. The effect of the situational response is scaled by the amount of perceived stress $\bar{s}$ that an agent is current experiencing.

The resulting equation for determining simulation parameters that depicting the agent's behaviors due to inherent personality traits and dynamically changing stress response is

$$
\text { SimParams }=S A_{p c} \mathbf{B}_{\text {stress }}+A_{a d j} \mathbf{P},
$$

where $\mathbf{P}$ is a vector representing an agents stable personality. When a stress is induced on an agent and the value of $S$ increases, the effect of $\mathbf{B}_{\text {stress }}$ will grow and thereby increase the stressful behaviors displayed by an agents. When the stress is removed, $S$ will decrease and the agents behavior will return to its stable personality $\mathbf{P}$ 


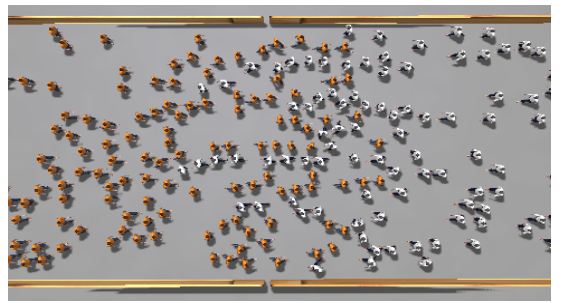

(a) Initial Approach

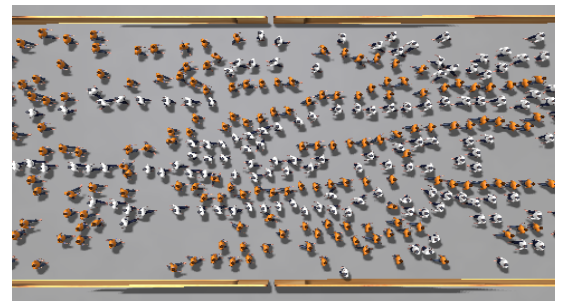

(b) Lane Formation

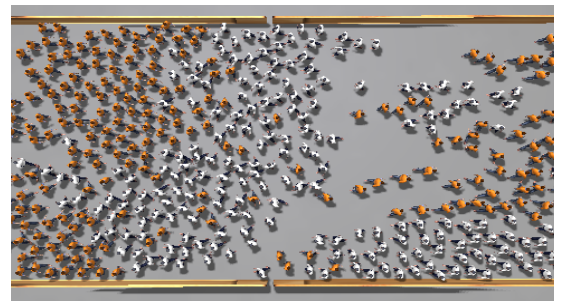

(c) Alarm Response

Figure 4: Opposing Group scenario. (a) Two opposing groups approach each other. (b) Agents initially form natural lanes. (c) After experiencing stress from the alarm the lane formation breaks down into uncooperative, clogged and congested behavior.

\subsection{Modeling from Real-world Data}

Eqn. 10 provides a complete description of how stress affects crowd behaviors in a multi-agent simulation. Given the definitions $A_{a d j}$, $S A_{p c}$, and $\mathbf{B}_{\text {stress }}$ from above, the free parameters to be determined for the model are $\alpha$ and $\beta$, which control how an agent responds to stress. One option is to let the simulation designer determine these parameters to create controlled or exaggerated crowd behaviors. However, they can also be found by fitting the stress response to real-word data.

Studies such as Crompton [1979] have found mappings between quantifiable changes behaviors, such as increasing speed, and quantifiable stressors such as the amount of time remaining on a pedestrian crossing signal. Matching the largest observed speeds for agents with little time left to cross provides a lower bound on the value of $\beta$. Likewise measuring the change in velocity over the time to cross the street will provide an estimate for $\alpha$. Finally for determining the form of the perceived stress function $\psi()$ we use a least-square power-law fitting.

Section 6.2 shows the result of this process on the data from [Crompton 1979]. In our simulations, we do not use the same value of $\alpha$ and $\beta$ for every agent, but rather sample them from a normal distribution centered at the values estimated from the data.

\section{Results}

We tested our method on several scenarios described below. For each scenario, we saw a variety of behaviors exhibited by the agents under stress, which matched the results from psychology literature both qualitatively and quantitatively.

Opposing Groups: Two large groups of agents move through a hallway in opposite directions. An alarm is triggered that provides an area stressor for the agents. See Figure 4.

Street Crossing: A single agent must cross the street with a time pressure stressor given by the crossing signal. See Figure 6 .

Chasing: An agent is being chased by a green monster. The chased agent experiences a positional stressor from the monster. See Figure $7 \mathrm{a}$.

Evacuation: One thousand agents must evacuate an office environment. All agents experience stress from the fire in the center of the building. Additionally some agents, shown in red shirts, also experience an interpersonal stressor from crowding. See Figure 5.

Shibuya Crossing: One thousand agents participate in a scrabble crossing modeled after the Shibuya metro station in Tokyo. The agents experience two stressors. The first is a pressure stressor when the crossing signal begins flashing, as a signal to clear the intersection. The second is a more intense area stress, when the crossing signal turns red indicating the vehicular traffic will resume soon. See Figure 1.

\subsection{Emergent Behaviors}

In the above scenarios agents exhibit several different behaviors commonly associated with increasing stress. These behaviors are not explicitly coded into the system, but rather emerge from the dynamic increase in aggressive and impulsive behavior that occurs from increasing the stress level.

A basic effect that can be seen in all of the scenarios is that agents will increase their speed as their stress increases. An example of this can be observed in the Evacuation scenario where agents near the fire run quickly to escape its spread. Likewise in the Shibuya Crossing scenario agents who are in the middle of the intersection increase their speed to a jog when the crossing light starting to flash and those who are still in the street when the light turns yellow or red switch to an all out run.

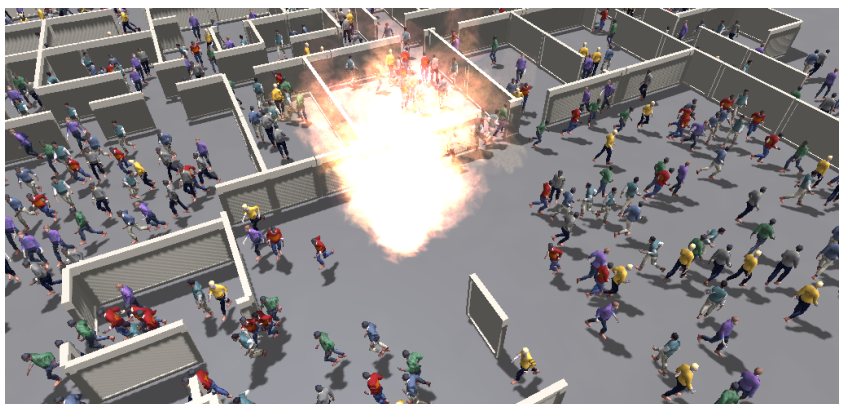

Figure 5: Evacuation scenario. Agents evacuate an office building in the presence of a fire stressor and crowding stressors.

Another stressed behavior displayed by agents is an increase in "selfish" behavior as stress increases. In the Evacuation scenario the agents who experience crowding stress have a higher level of stress than others who are only concerned with evacuating. The effect of this can be seen clearly in the accompanying video where these agents push their way out of the crowded doorway to exit faster than others. Likewise, in the Chasing scenario, the agent being chased cuts a near straight line through the crowd. In the same scenario, without a source of stress, the agent's path is deflected by the crowd of people it is trying to cut through (see Figure 7b).

Additionally, stressed agents show noticeably more impulsive or reckless behavior. In the Evacuation scenario stressed agents push and bump into each other as they crowd together to get through 


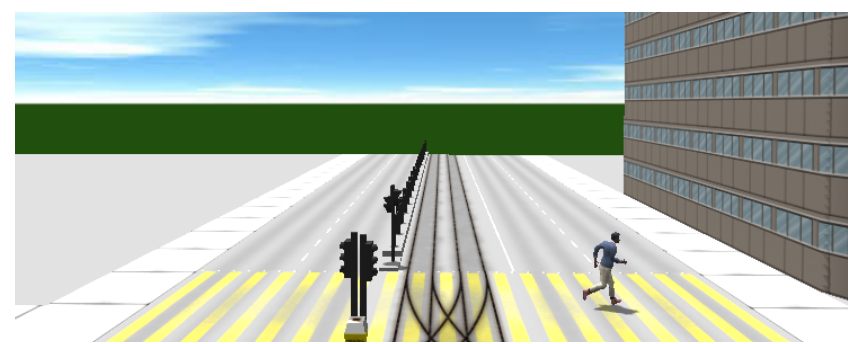

Figure 6: Street Crossing scenario. Agents cross a street after various delays. Agents who enter too late must run to cross in time.

the exit. Similarly, agents becoming increasingly stressed leads to the breakdown of the cooperative behavior which usually occurs between agents. This can be most clearly seen in the Opposing Groups scenario. Here, after the alarm sounds the natural lane formation that forms between the groups breaks down and the agents degenerate into a chaotic, inefficient flow (see Figure 4). When the alarm is turned off, and the stress subsides the agents eventually return to normal flow patterns.

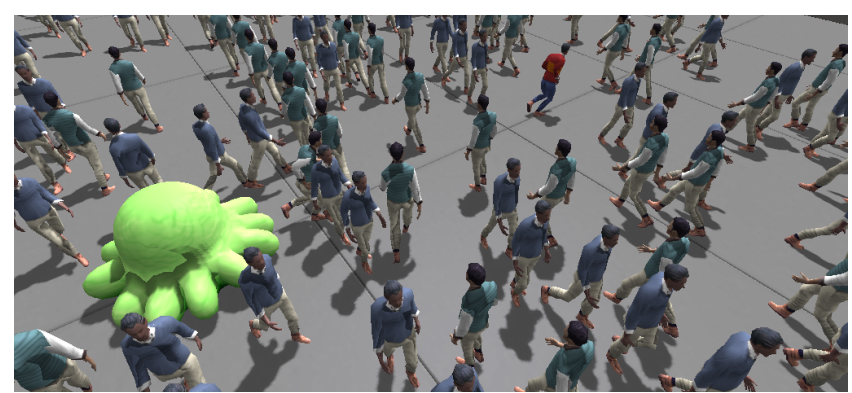

(a)

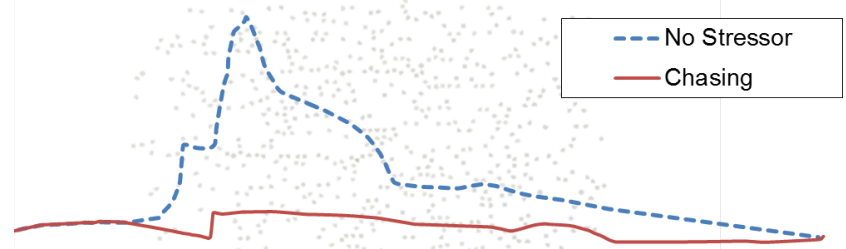

(b)

Figure 7: Chasing scenario. (a) A red shirted agent is chased through a crowd by a green monster causing a positional stressor. (b) If not being chased (dashed line), the agent's path drifts as it navigates through the crowd. With our model, the agent takes a faster, more direct path through the crowd (red line). This aggressive behavior is due to the effect of the stressor.

\subsection{Validation}

Due to the dynamic, incoherent nature of human crowds, preforming a precise validation of our method is a challenging problem. However, we can examine some quantitate and qualitative features of stress reported in the psychological literature and compare them to our results.

For example, in a study of pedestrians crossing roads, Crompton [1979] measured average crossing speed against how much a pedestrian has been delayed entering a street crossing from the start of the crossing signal. The study found that the later a pedestrian entered the street, the faster they walked. This effect can be seen with our method by presenting agents with a time pressure stressor with a $t_{\text {allowed }}$ for the time it takes to cross the street at an average walking speed. Using the method discussed in Sec. 5.3, we derived the values shown in Table 1 .

\begin{tabular}{|l|c|c|}
\hline Param & Value & Description \\
\hline$\alpha$ & .38 & Stress accumulation rate \\
$\beta$ & .80 & Maximum stress \\
$k$ & .012 & Power law coefficient \\
$n$ & 2 & Power law exponent \\
\hline
\end{tabular}

Table 1: Data-driven stress parameters. Values derived from fitting our stress model to street crossing data from Crompton [1979].

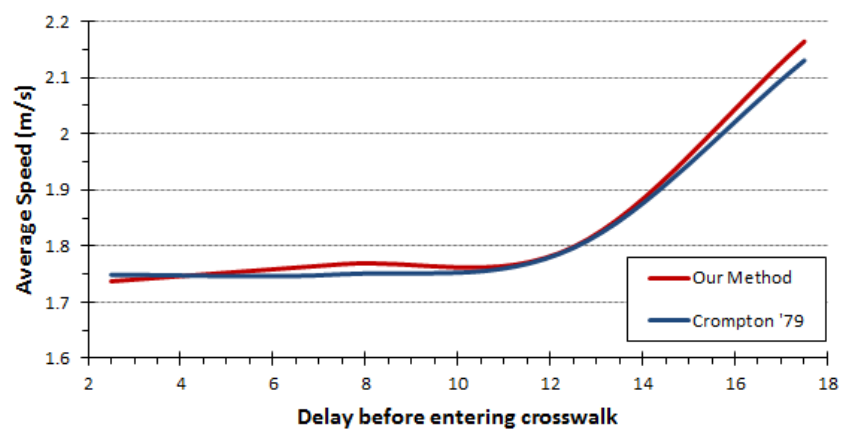

Figure 8: Comparison of simulated crossing speeds and real-world data. The less time left to cross, the faster agents move.

Another important result from the psychology of stress is known as the Yerkes-Dodson Law [Yerkes and Dodson 1908]. This is the observation that while for very simple tasks stress is usual beneficial to performance, when a task is sufficiently complicated too much stress causes a decrease in performance. We can see similar effects in our simulation. For example, in the Opposing Groups scenario, we can change the value of $\beta$ for the agents, changing the amount of stress they receive from the alarm. Here we measure performance as the average velocity of the agents over a small time window. With small amounts of stress agents velocities increase along with the aggressiveness of their behavior. However, when the stress level becomes too high, the lane formation breaks down causing agents to jam up and bump into each other, and slows down the overall progress. The "inverted U" shaped performance graph in Figure 9 is consistent with the Yerkes-Dodson Law.

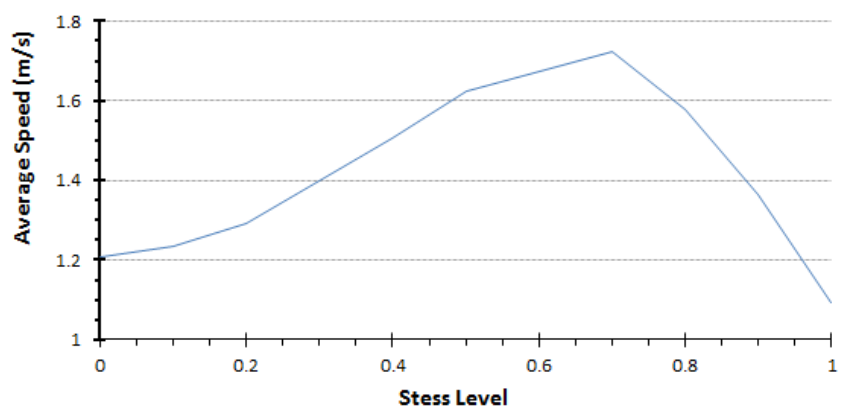

Figure 9: Average speed of agents in the Opposing Groups scenario at various levels of stress. The Yerkes-Dodson Law states that stress should increase performance up to a point then decrease it, a result which is matched by our model.

\subsection{Performance Results}

The Shibuya Crossing and Evacuation scenarios demonstrate the ability of our method to scale to large complex scenarios with thou- 
sands of agents and obstacles. Table 2 shows the execution time for simulating agents in several different scenarios. The timings were computed on a $3.2 \mathrm{GHz}$ Intel i7 processor. In all cases, the simulation ran at interactive rates.

\begin{tabular}{|l|c|c|c|}
\hline Scenario & $\begin{array}{c}\# \\
\text { Agents }\end{array}$ & $\begin{array}{c}\# \\
\text { Obstacles }\end{array}$ & $\begin{array}{c}\text { Time } \\
(\mathrm{ms})\end{array}$ \\
\hline Chasing & 602 & 0 & 18.8 \\
Opposing Groups & 800 & 2 & 17.2 \\
Evacuation & 1000 & 219 & 21.8 \\
Shibuya Crossing & 1000 & 205 & 20.6 \\
\hline
\end{tabular}

Table 2: Performance timings per frame.

\section{Conclusion}

We have introduced a method for modeling the dynamic changes in behavior that result from situational factors. Our method derives a linearized approximation of the well-established theory of Generalized Adaptation Syndrome for modeling stress response. Our approach is able to match quantitative studies of human behavior, reproduce important phenomena such as the Yerkes-Dodson Law, and display a variety of emergent dynamic behaviors, all at interactive rates.

There are, however, some limitations to our approach. We have made a few simplifying assumptions, and have limited our application to short-term stressors ignoring the effects exhaustion or death. Additionally, we do not address how the stress response should interact with the crowd rendering system (e.g. changing the facial expression of agents under stress). We would like to investigate these areas in future work. Additionally we would like to integrate our system in a video game engine to allow for stressors to arise from user interaction.

\section{Acknowledgements}

We are grateful to the reviewers for their comments, We would like to thank Sean Curtis and Jamie Snape for their help. This research is supported in part by ARO Contract W911NF-04-1-0088, NSF awards 0917040, 0904990, 100057 and 1117127, and Intel.

\section{References}

Anderson, C. A. 2001. Heat and violence. Current Directions in Psychological Science 10, 1, pp. 33-38.

BERKOWITZ, L. 1990. On the formation and regulation of anger and aggression: A cognitive-neoassociationistic analysis. American Psychologist 45, 4, 494 - 503.

CATTELL, R. 1952. Factor analysis: an introduction and manual for the psychologist and social scientist. Harper's psychological series. Harper.

Cox, T. 1978. Stress. Macmillan.

CROMPTON, D. 1979. Pedestrian delay, annoyance and risk: preliminary results from a 2 years study. In Proceedings of PTRC Summer Annual Meeting, 275-299.

Dobbyn, S., Hamill, J., O’Conor, K., And O'Sullivan, C. 2005. Geopostors: a real-time geometry / impostor crowd rendering system. In Interactive $3 D$ Graphics and games, 95-102.

Durupinar, F., Pelechano, N., Allbeck, J., Gu anddu andkbay, U., And BADLER, N. 2011. How the ocean personality model affects the perception of crowds. Computer Graphics and Applications, IEEE 31, 3 (may-june), 22 -31.

ERIKSEN, H. R., OlfF, M., Murison, R., AND Ursin, H. 1999. The time dimension in stress responses: relevance for survival and health. Psychiatry Research 85 , $1,39-50$.

Evans, G. 1984. Environmental Stress. Cambridge University Press.

EYSENCK, M. 2002. Simply psychology. Psychology Press.
GuY, S. J., Kim, S., Lin, M. C., And Manocha, D. 2011. Simulating heterogeneous crowd behaviors using personality trait theory. In Symposium on Computer Animation, ACM, 43-52.

HEIDER, F. 1982. The psychology of interpersonal relations. Lawrence Erlbaum Associates.

K.B., AND RASMUSSEN. 1979. Annoyance from simulated road traffic noise. Journal of Sound and Vibration 65, 2, $203-214$.

LAZARUS, R. S. 1993. From Psychological Stress to the Emotions: A History of Changing Outlooks. Annual Reviews Psychology 44, 1-22.

LEON, J. 2010. Pedestrian psychology and safety. http://www.drdriving.org/pedestrians/.

Middlemist, R. D., Knowles, E. S., And Matter, C. F. 1976. Personal space invasions in the lavatory: Suggestive evidence for arousal. Journal of Personality and Social Psychology 33, 5, $541-546$.

Miller, N. E. 1941. I. the frustration-aggression hypothesis. Psychological Review $48,4,337-342$.

MordKoff, A. M. 1964. The relationship between psychological and physiological response to stress. Psychosomatic Medicine 26, 2, 135-150.

Nuria Pelechano, Kevin O'Brien, B. S. N. B. 2005. Crowd simulation incorporating agent psychological models, roles and communication. In First International Workshop on Crowd Simulation. (V-Crowds '05), 21-30.

NyGREN, M., 2007. Simulation of human behaviour in stressful crowd situations.

OSWALD, AND BRATFISCH. 1969. A further study of the relation between subjective distance and emotional involvement. Acta Psychologica 29, 0, 244-255.

Patil, S., VAn den Berg, J., Curtis, S., Lin, M., And Manocha, D. 2011. Directing crowd simulations using navigation fields. Visualization and Computer Graphics, IEEE Transactions on 17, 2 (feb.), 244-254.

Pelechano, N., Allbeck, J. M., And Badler, N. I. 2007. Controlling individual agents in high-density crowd simulation. In Symposium on Computer animation, 99-108.

Pelechano, N., Allbeck, J., And Badler, N. 2008. Virtual crowds: Methods, simulation, and control. Synthesis Lectures on Computer Graphics and Animation $3,1,1-176$.

ReYnolds, C. 1999. Steering Behaviors for Autonomous Characters. In Game Developers Conference 1999.

Sakuma, T., Mukai, T., And Kuriyama, S. 2005. Psychological model for animating crowded pedestrians: Virtual humans and social agents. Comput. Animat. Virtual Worlds 16 (July), 343-351.

SElye, H. 1956. The stress of life. No. v. 5 in McGraw-Hill paperbacks. McGraw-Hill.

Shao, W., And Terzopoulos, D. 2005. Autonomous pedestrians. In Symposium on Computer animation, 19-28.

Shopf, J., Barczak, J., OAT, C., And TATARchuk, N. 2008. March of the froblins: simulation and rendering massive crowds of intelligent and detailed creatures on gpu. In ACM SIGGRAPH 2008 classes, 52-101.

Stevens, S. S. 1957. On the psychophysical law. Psychological Review 64, 3, 153 181.

Teghtsoonian, R., And Frost, R. O. 1982. The effects of viewing distance on fear of snakes. Journal of Behavior Therapy and Experimental Psychiatry 13, 3, $181-190$

VAn Den Berg, J., GuY, S. J., Lin, M., And Manocha, D. 2011. Reciprocal n-body collision avoidance. In Robotics Research: The 14th International Symposium ISRR, Springer Tracts in Advanced Robotics (STAR), vol. 70, 3-19.

Yeh, H., Curtis, S., Patil, S., van den Berg, J., Manocha, D., And Lin, M. 2008. Composite agents. In Symposium on Computer Animation, 39-47.

YeRKES, R. M., AND Dodson, J. D. 1908. The relation of strength of stimulus to rapidity of habit-formation. Journal of Comparative Neurology and Psychology $18,5,459-482$.

Yersin, B., MaÏm, J., Pettré, J., And Thalmann, D. 2009. Crowd patches: populating large-scale virtual environments for real-time applications. In Interactive $3 D$ graphics and games, 207-214.

YU, Q., AND TeRzopoulos, D. 2007. A decision network framework for the behavioral animation of virtual humans. In Symposium on Computer animation, 119 128. 